\title{
Sarcopenia does not worsen survival in patients with cirrhosis undergoing transjugular intrahepatic portosystemic shunt for refractory ascites
}

Amine Benmassaoud, MD ${ }^{1,2}$; Davide Roccarina, $\mathrm{MD}^{1,2}$; Francesco Arico ${ }^{1,2}$; Gioacchino Leandro, $\mathrm{MD}^{3}$;Becky Yu ${ }^{1,2}$; Felix Cheng ${ }^{1,2}$; Dominic Yu, MD ${ }^{4}$; David Patch, MD ${ }^{1,2}$; Emmanuel A. Tsochatzis, $\mathrm{PhD}^{1,2}$.

${ }^{1}$ The Royal Free Sheila Sherlock Liver Centre, Royal Free London NHS Trust, London, UK

${ }^{2}$ UCL Institute for Liver and Digestive Health, University College of London, London, UK

${ }^{3}$ National Institute of Gastroenterology, S. De Bellis Research Hospital, Castellana Grotte, Italy

${ }^{4}$ Department of Radiology, Royal Free London NHS Trust, London, UK

\section{Corresponding Author}

Dr. Emmanuel Tsochatzis, PhD

Sheila Sherlock Liver Unit and UCL Institute for Liver and Digestive Health

Royal Free Hospital and UCL,

Pond Street,

London, NW3 2QG, UK

Email: e.tsochatzis@ucl.ac.uk 
Guarantor of the article

Dr. Emmanuel Tsochatzis

\section{Specific author contributions}

$A B$ and $E T$ planned the study; $A B, D R, F A, B Y$, and FC collected the data; $A B, G L, D Y, D P$, and ET interpreted data; $A B, D P$, and $E T$ drafted the manuscript. $A B, D R, F A, G L, B Y, F C, D Y, D P$ and $E T$ have approved the final draft of the manuscript.

\section{Financial support}

This study did not receive any financial support.

\section{Potential competing interests}

$A B, D R, F A, G L, B Y, F C, D Y, D P$ and $E T$ have no conflict of interest to disclose

Word count: 999/1000

\section{Keywords}

Skeletal Muscle Index, Psoas Muscle Index, Hepatic Encephalopathy, Liver transplantation, Malnutrition 


\section{Study Highlights}

\section{WHAT IS KNOWN}

Sarcopenia is associated with increased mortality in patients with cirrhosis

The impact of sarcopenia in patients undergoing TIPSS insertion for refractory ascites is unknown WHAT IS NEW

Sarcopenia is not associated with worse outcome after TIPSS insertion for refractory ascites

Sarcopenia is not associated with increased incidence of encephalopathy following TIPSS insertion for refractory ascites

Insertion of TIPSS in patients with refractory ascites might lead to improvement in muscle mass 
ABSTRACT

Objectives

The impact of sarcopenia in patients undergoing transjugular intrahepatic portosystemic shunt (TIPSS) insertion for refractory ascites is unknown.

\section{Methods}

All adult patients who underwent TIPSS insertion for refractory ascites between 2010 and 2018 were included. Skeletal muscle index at L3 was used to determine sarcopenia status.

\section{Results}

107 patients were followed for 14.2 months. Sarcopenia was present in $57 \%$ of patients. No patient had history of pre-TIPSS hepatic encephalopathy (HE). De novo HE occurred in $30 \%$ of patients. On MVA, only platelet count and L3-SMI predicted de novo HE. On MVA, age and MELD-Na predicted mortality whereas L3-SMI and sarcopenia did not. In patients with repeat imaging, L3-SMI improved significantly post-TIPSS compared to baseline.

\section{Conclusions}

Sarcopenia should not be considered as a contra-indication to TIPSS insertion in refractory ascites as it is not associated with de novo HE or increased mortality. 
Word count: $142 / 150$

\section{Keywords}

Skeletal Muscle Index, Psoas Muscle Index, Hepatic Encephalopathy, Liver transplantation, malnutrition 


\section{INTRODUCTION}

The insertion of a transjugular intrahepatic portosystemic shunt (TIPSS) has become an effective treatment for refractory ascites in selected patients, with recent data showing improved 1-year transplant free survival (1-5). Sarcopenia, defined as skeletal muscle index (L3-SMI) or total psoas muscle index (L3-PMI) at the third lumbar vertebrae, is a predictor of poor outcome in patients with cirrhosis before and after transplantation(6-9). We investigated the impact of pre-existing sarcopenia, by L3-SMI or L3-PMI, on incidence of de novo hepatic encephalopathy (HE), and overall mortality post-TIPSS in a large cohort of consecutive patients who underwent TIPSS insertion for refractory ascites $(10,11)$.

\section{METHODS}

We conducted a retrospective cohort study including all adult patients with cirrhosis who underwent TIPSS insertion for refractory ascites and had baseline cross-sectional abdominal imaging within 6 months before or 2 weeks after TIPSS insertion at our institution between April 01/04/2010 and 01/04/2018. To be considered for TIPSS insertion, patients required repeated large volume paracentesis for at least six months. As per our standard protocol, patients with a prior diagnosis of HE or abnormal EEG were not considered for TIPSS. Patients were identified through a prospective database. Ethics approval was waived as per standard procedure for retrospective studies which fall under the audit category. 


\section{Sarcopenia assessment}

To measure L3-SMI and L3-PMI at baseline, transverse sections of cross-sectional imaging were analyzed using ImageJ (version 1.51, Wayne Rasband, National Institutes of Health)(6). Previously validated for L3-SMI, a MRI-specific method was employed when necessary (12). All measurements were performed by a single trained physician in a blinded manner. Patients in the lowest quartile of L3-PMI as stratified by sex were considered to be sarcopenic(6). Women and men with L3-SMI under $39 \mathrm{~cm}^{2} / \mathrm{m}^{2}$ and $50 \mathrm{~cm}^{2} / \mathrm{m}^{2}$, respectively, were considered to be sarcopenic(8).

\section{Outcome measures}

Patients were followed by their treating hepatologist and were seen at least every 6 months. Occurrence of de novo $\mathrm{HE}$, defined as clinically evident or at least grade II as per West Haven criteria, was recorded as well as its treatment (13). Liver transplantation and death were recorded.

\section{Statistical analysis}

Association between risk factors and de novo HE or survival was assessed using multivariate Cox regression reporting hazard ratio (HR). Competing risk analysis was performed for survival considering liver transplantation as a competing event. Statistical analysis was carried using SPSS (version 25.0, IBM, New York, NY, USA) and STATA (version 16, STATA Corp LLC, College Station, TX, USA). 


\section{RESULTS}

\section{Main characteristics}

A total of 165 patients underwent TIPSS insertion for refractory ascites during the study period. After application of inclusion and exclusion criteria, the final cohort was constituted of 107 patients. Baseline characteristics are presented in Table 1. L3-PMI results are presented in supplemental analysis (Supplemental table 1-5). Multivariate logistic regression showed that BMI and sex were associated with baseline sarcopenia.

\section{Change of muscle mass during follow-up}

Follow-up cross sectional imaging was available in 41 patients. The median time to the last crosssectional imaging was 12.7 (IQR 19.3) months. In this subset of patients, L3-SMI increased from $45.2 \pm 9.0 \mathrm{~cm}^{2} / \mathrm{m}^{2}$ to $48.4 \pm 11.5 \mathrm{~cm}^{2} / \mathrm{m}^{2}$ at last imaging $(p=0.009)$. The improvement was more significant in women where L3-SMI increased from $41.3 \pm 7.7 \mathrm{~cm}^{2} / \mathrm{m}^{2}$ to $44.0 \pm 9.7 \mathrm{~cm}^{2} / \mathrm{m}^{2}$ $(p=0.013)$ compared to $47.9 \pm 9.1 \mathrm{~cm}^{2} / \mathrm{m}^{2}$ to $51.5 \pm 11.9 \mathrm{~cm}^{2} / \mathrm{m}^{2}(p=0.07)$ in men.

\section{Hepatic Encephalopathy}

Overall, 32 (29.9\%) patients suffered from de novo HE following TIPSS insertion. Medical therapy with lactulose or combination with rifaximin controlled HE in 27 (84.3\%) patients, whereas 4 (12.5\%) patients needed TIPSS reduction and 1 (3.1\%) TIPSS occlusion. Multivariate cox regression analysis showed that platelets and L3-SMI were associated with de novo HE while sarcopenia was not (Table 2). 


\section{Mortality}

Overall, 34 (31.8\%) patients died and 18 (16.8\%) were transplanted during follow-up. The 1, 3, and 5 -year overall survival rates were $79 \%, 56 \%$, and $36 \%$ respectively. Multivariate analysis presented in table 4 shows that age, MELD-Na and platelet count were independent predictors of mortality, while L3-SMI and sarcopenia were not. Finally, in a competing risk analysis with liver transplantation as a competing event, L3-SMI and sarcopenia were not associated with mortality (Table 3).

\section{DISCUSSION}

This is the first study that assessed the impact of sarcopenia on patients undergoing TIPSS insertion for refractory ascites. Although a higher L3-SMI was associated with a lower risk of de novo $\mathrm{HE}$, sarcopenia was not. Furthermore, in patients with available repeat cross-sectional imaging, we noted an improvement of L3-SMI over time. We also demonstrated that neither L3SMI nor L3-PMI were able to predict mortality following TIPSS insertion for refractory ascites.

A recent study concluded that sarcopenia was associated with higher mortality post-TIPSS(12). This particular study spanned 20 years starting in 1993 and therefore included patients with uncovered stents. It also included patients who received TIPSS for various indications and $60 \%$ of patients at inclusion had HE (12). At this stage, we believe that our study provides a more accurate depiction of patients undergoing TIPSS insertion for refractory ascites and that baseline sarcopenia should not be seen as a contraindication. 
De novo HE occurred in $30 \%$ of patients, consistent with previous cohorts $(4,5,14)$. Nardelli et al. identified sarcopenia as a risk factor for post-TIPSS HE(11). In their cohort, they had a higher than expected incidence of HE which could be due to the use of larger stents (15). In our cohort, the

most commonly used stents measured $8 \mathrm{~mm}$. Furthermore, our rates of post-TIPSS HE might have been influenced by our patient selection as no patient with pre-existing HE received a TIPSS.

Our study has several strengths as it provides an updated real-world look at the outcomes of a large cohort of patients with refractory ascites treated with TIPSS to minimize confounding by indication. Furthermore, we used two separate methods to assess sarcopenia, namely L3-SMI and L3-PMI $(6,8)$, not showing a difference. Our study has limitations inherent to its retrospective nature. An additional limitation is that we did not evaluate patients for minimal HE.

In conclusion, the presence of sarcopenia at baseline should not be seen as a contra-indication to TIPSS insertion in patients with cirrhosis and refractory ascites.

\section{Acknowledgements}

Dr. Amine Benmassaoud was supported by an educational grant from the Canadian Association for the Study of the Liver. 


\section{REFERENCES}

1. Ferral H, Bjarnason H, Wegryn SA, Rengel GJ, Nazarian GK, Rank JM, et al. Refractory ascites: early experience in treatment with transjugular intrahepatic portosystemic shunt. Radiology. 1993;189(3):795-801.

2. Rossle M, Ochs A, Gulberg V, Siegerstetter V, Holl J, Deibert P, et al. A comparison of paracentesis and transjugular intrahepatic portosystemic shunting in patients with ascites. $\mathrm{N}$ Engl J Med. 2000;342(23):1701-7.

3. Gines P, Uriz J, Calahorra B, Garcia-Tsao G, Kamath PS, Del Arbol LR, et al. Transjugular intrahepatic portosystemic shunting versus paracentesis plus albumin for refractory ascites in cirrhosis. Gastroenterology. 2002;123(6):1839-47.

4. Bureau C, Thabut D, Oberti F, Dharancy S, Carbonell N, Bouvier A, et al. Transjugular Intrahepatic Portosystemic Shunts With Covered Stents Increase Transplant-Free Survival of Patients With Cirrhosis and Recurrent Ascites. Gastroenterology. 2017;152(1):157-63.

5. Narahara Y, Kanazawa H, Fukuda T, Matsushita Y, Harimoto H, Kidokoro H, et al.

Transjugular intrahepatic portosystemic shunt versus paracentesis plus albumin in patients with refractory ascites who have good hepatic and renal function: a prospective randomized trial. J Gastroenterol. 2011;46(1):78-85.

6. Kalafateli M, Mantzoukis K, Choi Yau Y, Mohammad AO, Arora S, Rodrigues S, et al. Malnutrition and sarcopenia predict post-liver transplantation outcomes independently of the Model for End-stage Liver Disease score. J Cachexia Sarcopenia Muscle. 2017;8(1):113-21.

7. Moctezuma-Velazquez C, Ebadi M, Bhanji RA, Stirnimann G, Tandon P, Montano-Loza AJ. Limited performance of subjective global assessment compared to computed tomographydetermined sarcopenia in predicting adverse clinical outcomes in patients with cirrhosis. Clin Nutr. 2018.

8. Carey EJ, Lai JC, Wang CW, Dasarathy S, Lobach I, Montano-Loza AJ, et al. A multicenter study to define sarcopenia in patients with end-stage liver disease. Liver Transpl. 2017;23(5):625-33.

9. Montano-Loza AJ, Meza-Junco J, Prado CM, Lieffers JR, Baracos VE, Bain VG, et al. Muscle wasting is associated with mortality in patients with cirrhosis. Clin Gastroenterol Hepatol. 2012;10(2):166-73, 73 e1.

10. Tsien C, Shah SN, McCullough AJ, Dasarathy S. Reversal of sarcopenia predicts survival after a transjugular intrahepatic portosystemic stent. Eur J Gastroenterol Hepatol. 2013;25(1):85-93.

11. Nardelli S, Lattanzi B, Torrisi S, Greco F, Farcomeni A, Gioia S, et al. Sarcopenia Is Risk Factor for Development of Hepatic Encephalopathy After Transjugular Intrahepatic Portosystemic Shunt Placement. Clin Gastroenterol Hepatol. 2017;15(6):934-6.

12. Praktiknjo M, Book M, Luetkens J, Pohlmann A, Meyer C, Thomas D, et al. Fat-free muscle mass in magnetic resonance imaging predicts acute-on-chronic liver failure and survival in decompensated cirrhosis. Hepatology. 2018;67(3):1014-26.

13. Vilstrup H, Amodio P, Bajaj J, Cordoba J, Ferenci P, Mullen KD, et al. Hepatic encephalopathy in chronic liver disease: 2014 Practice Guideline by the American Association 
for the Study of Liver Diseases and the European Association for the Study of the Liver. Hepatology. 2014;60(2):715-35.

14. Salerno F, Camma C, Enea M, Rossle M, Wong F. Transjugular intrahepatic portosystemic shunt for refractory ascites: a meta-analysis of individual patient data. Gastroenterology. 2007;133(3):825-34.

15. Wang $Q$, Lv Y, Bai M, Wang Z, Liu H, He C, et al. Eight millimetre covered TIPS does not compromise shunt function but reduces hepatic encephalopathy in preventing variceal rebleeding. J Hepatol. 2017;67(3):508-16. 
TABLES

Table 1. Baseline characteristics of patients divided by sarcopenia using L3-SMI.

\begin{tabular}{|c|c|c|c|c|}
\hline & $\begin{array}{l}\text { All patients } \\
(\mathrm{n}=107)\end{array}$ & $\begin{array}{l}\text { Sarcopenia } \\
(n=61)\end{array}$ & $\begin{array}{l}\text { No Sarcopenia } \\
(n=46)\end{array}$ & $p$-value \\
\hline Age, years, SD & $55.3(9.7)$ & $54.9(10.3)$ & $55.8(9.0)$ & 0.634 \\
\hline Sex, male $n(\%)$ & $65(60.7)$ & $45(73.8)$ & $20(43.5)$ & 0.003 \\
\hline Follow-up, months, IQR & $14.2(31.8)$ & $15.7(29.5)$ & $13.9(43.7)$ & 0.283 \\
\hline Etiology, n(\%) & & & & 0.091 \\
\hline $\mathrm{HCV}$ & $12(11.2)$ & $6(9.8)$ & $6(13.0)$ & \\
\hline ALD & $69(64.5)$ & $43(70.5)$ & $26(56.5)$ & \\
\hline NAFLD & $13(12.1)$ & $3(4.9)$ & $10(21.7)$ & \\
\hline Cryptogenic & $4(3.7)$ & $3(4.9)$ & $1(2.2)$ & \\
\hline Other & $9(8.4)$ & $6(9.8)$ & $3(6.5)$ & \\
\hline HCV SVR, n(\%) & $9(75.0)$ & $5(83.3)$ & $4(66.7)$ & 1.000 \\
\hline EtOH Abstinence, $n(\%)$ & $49(76.6)$ & $28(71.8)$ & $21(84.0)$ & 0.368 \\
\hline Hemodynamic Target, n(\%) & $92(94.8)$ & 51 (91.1) & $41(100)$ & 0.071 \\
\hline Stent Diameter, $\mathrm{n}(\%)$ & & & & 0.416 \\
\hline $8 \mathrm{~mm}$ & $66(63.5)$ & $39(67.2)$ & $27(58.7)$ & \\
\hline $10 \mathrm{~mm}$ & 38 (36.5) & $19(32.8)$ & 19 (41.3) & \\
\hline Gradient pre-TIPS, mmHg, IQR & $14(7)$ & $15(6)$ & $13(8)$ & 0.373 \\
\hline Gradient post-TIPS, $\mathrm{mmHg}$, IQR & $8(4)$ & $9(5)$ & $8(3)$ & 0.596 \\
\hline Child Pugh Score, IQR & $8(2)$ & $8(2)$ & $8(2)$ & 0.768 \\
\hline$B, n(\%)$ & $96(89.7)$ & $56(91.8)$ & 40 (87.0) & \\
\hline $\mathrm{C}, \mathrm{n}(\%)$ & $11(10.3)$ & $5(8.2)$ & $6(13.0)$ & \\
\hline Hepatic Encephalopathy, n (\%) & $0(0)$ & $0(0)$ & $0(0)$ & 1.000 \\
\hline $\mathrm{BMI}, \mathrm{kg} / \mathrm{m}^{2}, \mathrm{IQR}$ & $22.1(6.2)$ & $21.1(5.0)$ & $24.3(6.7)$ & 0.001 \\
\hline Platelets, $\times 10^{9} / \mathrm{L}$, IQR & $129(108)$ & $147(135)$ & $116(89)$ & 0.355 \\
\hline INR, IQR & $1.3(0.3)$ & $1.3(0.3)$ & $1.3(0.3)$ & 0.755 \\
\hline Serum sodium, mmol/L, SD & $135(5)$ & $135(4)$ & $136(5)$ & 0.061 \\
\hline Serum creatinine, $\mu \mathrm{mol} / \mathrm{L}, \mathrm{IQR}$ & $77(39)$ & $78(42)$ & $76(40)$ & 0.930 \\
\hline Serum bilirubin, $\mu \mathrm{mol} / \mathrm{L}, \mathrm{IQR}$ & $18(22)$ & $18(21)$ & $18(24)$ & 0.932 \\
\hline Serum albumin, $g / L, S D$ & $34(5)$ & $34(5)$ & $34(5)$ & 0.967 \\
\hline MELD-Na, IQR & $11(8)$ & $11(8)$ & $12(6)$ & 0.882 \\
\hline L3-SMI, $\mathrm{cm}^{2} / \mathrm{m}^{2}$ IQR & $44.0(12.0)$ & $41.0(10.4)$ & $51.7(15.7)$ & $<0.001$ \\
\hline L3-PMI, $\mathrm{mm}^{2} / \mathrm{m}^{2}, \mathrm{SD}$ & $510.4(216.8)$ & $475.5(141.9)$ & $632.2(193.9)$ & $<0.001$ \\
\hline
\end{tabular}

Legend: Categorical variables are expressed as numbers (\%), continuous variables with a non-normal distribution are expressed as median (IQR), and continuous variables with a normal distribution are expressed as mean (SD), unless otherwise specified. ALD, alcohol-related liver disease; BMI, body mass index; HCV, hepatitis C virus; IQR, interquartile range; INR, international normalized ratio; L3-PMI, total psoas muscle area indexed for height at the third lumbar vertebrae; L3-SMI, skeletal muscle area indexed for height at the third lumbar vertebrae; MELD-Na, model for end stage liver disease with sodium; NAFLD, non-alcoholic fatty liver disease; SD, standard deviation; SVR, sustained virological response; TIPSS, transjugular intrahepatic portosystemic shunt. 
Table 2. Predictors of de novo hepatic encephalopathy following TIPSS insertion with sarcopenia defined using L3-SMI.

\begin{tabular}{|l|l|l|l|l|}
\hline & \multicolumn{2}{|l|}{ Univariate cox regression analysis } & \multicolumn{2}{l|}{ Multivariate cox regression analysis } \\
\hline Variables & $\mathrm{HR}(95 \% \mathrm{Cl})$ & $\mathrm{p}$-value & $\mathrm{HR}(95 \% \mathrm{Cl})$ & $\mathrm{p}$-value \\
\hline Age, per year & $1.00(0.97-1.04)$ & 0.95 & - & - \\
\hline Male sex & $0.66(0.32-1.37)$ & 0.27 & - & - \\
\hline BMI, per kg/m² & $0.97(0.90-1.05)$ & 0.41 & - & - \\
\hline Platelets & $\mathbf{0 . 9 9}(\mathbf{0 . 9 9 - 1 . 0 0 )}$ & $\mathbf{0 . 0 1}$ & $\mathbf{0 . 9 9}(\mathbf{0 . 9 9 - 1 . 0 0 )}$ & $<$ \\
\hline INR & $2.05(0.68-6.22)$ & 0.21 & - & - \\
\hline Serum creatinine & $1.00(1.00-1.01)$ & 0.68 & - & - \\
\hline Serum bilirubin & $\mathbf{1 . 0 2 ( 1 . 0 0 - 1 . 0 4 )}$ & $\mathbf{0 . 0 2}$ & - & - \\
\hline Serum albumin & $1.04(0.97-1.10)$ & 0.29 & - & - \\
\hline MELD-Na & $1.06(0.99-1.14)$ & 0.08 & $1.01(0.99-1.03)$ & 0.25 \\
\hline Child-Pugh Score & $1.09(0.77-1.53)$ & 0.63 & - & - \\
\hline L3-SMI, per cm ${ }^{2} / \mathrm{m}^{2}$ & $\mathbf{0 . 9 6}(\mathbf{0 . 9 2 - 1 . 0 0 )}$ & $\mathbf{0 . 0 3}$ & $\mathbf{0 . 9 5}(\mathbf{0 . 9 1}-\mathbf{0 . 9 9})$ & $\mathbf{0 . 0 1}$ \\
\hline Sarcopenia ${ }^{\text {L3-SMI }}$ & $1.77(0.85-3.67)$ & 0.13 & - & - \\
\hline
\end{tabular}

Legend: $\mathrm{BMI}$, body mass index; $\mathrm{Cl}$, confidence interval; $\mathrm{HR}$, hazard ratio; INR, international normalized ratio; L3SMI, skeletal muscle area indexed for height at the third lumbar vertebrae; MELD, model for end stage liver disease; TIPSS, transjugular intrahepatic portosystemic shunt. ${ }^{\S}$ Multivariate analysis was conducted using variables with a $p$-value $<0.1$ in the univariate analysis. As a consequence, platelet count and MELD-Na were the only variables entered in the MVA. MELD-Na was chosen over its individual components. 
Table 3. Predictors of mortality following TIPSS insertion with sarcopenia defined using L3-SMI.

\begin{tabular}{|c|c|c|c|c|c|c|}
\hline \multirow[b]{2}{*}{ Variables } & \multicolumn{2}{|c|}{$\begin{array}{l}\text { Univariate Cox } \\
\text { regression analysis }\end{array}$} & \multicolumn{2}{|c|}{$\begin{array}{l}\text { Multivariate Cox } \\
\text { regression analysis }{ }^{\S}\end{array}$} & \multicolumn{2}{|c|}{$\begin{array}{l}\text { Multivariate Cox } \\
\text { regression competing risk }\end{array}$} \\
\hline & $\mathrm{HR}(95 \% \mathrm{Cl})$ & $p$-value & $\mathrm{HR}(95 \% \mathrm{Cl})$ & $p$-value & SHR $(95 \% \mathrm{Cl})$ & $p$-value \\
\hline Age, per year & $1.04(1.00-1.07)$ & 0.05 & $1.04(1.01-1.08)$ & 0.02 & $1.04(1.01-1.07)$ & $<0.01$ \\
\hline Male sex & $1.05(0.53-2.07)$ & 0.89 & - & - & - & - \\
\hline $\mathrm{BMI}$, per $\mathrm{kg} / \mathrm{m}^{2}$ & $1.02(0.96-1.08)$ & 0.61 & - & - & - & - \\
\hline Platelets & $0.99(0.99-1.00)$ & 0.02 & $0.99(0.99-1.00)$ & 0.05 & $0.99(0.99-1.00)$ & 0.02 \\
\hline INR & $1.38(0.42-4.51)$ & 0.60 & - & - & - & - \\
\hline Serum creatinine & $1.00(1.00-1.01)$ & 0.17 & - & - & - & - \\
\hline Serum bilirubin & $1.02(1.00-1.04)$ & 0.04 & - & - & - & - \\
\hline Serum albumin & $0.99(0.93-1.06)$ & 0.83 & - & - & - & - \\
\hline MELD-Na & $1.09(1.02-1.16)$ & 0.01 & $1.08(1.00-1.16)$ & 0.04 & - & - \\
\hline Child-Pugh Score & $1.21(0.87-1.66)$ & 0.25 & - & - & - & - \\
\hline L3-SMI,per $\mathrm{cm}^{2} / \mathrm{m}^{2}$ & $0.99(0.96-1.03)$ & 0.70 & & & - & - \\
\hline Sarcopenia ${ }^{\text {L3-SMI }}$ & $0.82(0.42-1.62)$ & 0.57 & - & - & - & - \\
\hline
\end{tabular}

Legend: $\mathrm{BMI}$, body mass index; $\mathrm{Cl}$, confidence interval; HR, hazard ratio; INR, international normalized ratio; L3SMI, skeletal muscle area indexed for height at the third lumbar vertebrae; MELD, model for end stage liver disease; SHR, subdistribution hazard ratio. ${ }^{\S}$ Multivariate analysis was conducted using variables with a $p$-value < 0.1 in the univariate analysis. As a consequence, age, platelet count and MELD-Na were the only variables entered in the MVA. MELD-Na was chosen over its individual components. ${ }^{\circ}$ Multivariate analysis conducted using competing risk analysis. 
Supplemental Table 1. Baseline characteristics of patients divided by presence of baseline imaging.

\begin{tabular}{|c|c|c|c|c|}
\hline & $\begin{array}{l}\text { All patients } \\
(n=165)\end{array}$ & $\begin{array}{l}\text { Without imaging } \\
(n=58)\end{array}$ & $\begin{array}{l}\text { With imaging } \\
(n=107)\end{array}$ & $p$-value \\
\hline Age, years, SD & $56.2(10.0)$ & $57.7(10.5)$ & $55.3(9.7)$ & 0.15 \\
\hline Sex, male n(\%) & $98(59.4)$ & $33(56.9)$ & $65(60.7)$ & 0.74 \\
\hline Follow-up, months, IQR & $11.5(26.0)$ & $8.9(24.9)$ & $14.2(31.8)$ & 0.05 \\
\hline Etiology, $\mathrm{n}(\%)$ & & & & 0.78 \\
\hline $\mathrm{HCV}$ & $17(10.3)$ & $5(8.6)$ & $12(11.2)$ & \\
\hline ALD & $107(64.8)$ & $38(65.5)$ & $69(64.5)$ & \\
\hline NAFLD & $18(10.9)$ & $5(8.6)$ & $13(12.1)$ & \\
\hline Cryptogenic & $6(3.6)$ & $2(3.4)$ & $4(3.7)$ & \\
\hline Other & $17(10.3)$ & $8(13.8)$ & $9(8.4)$ & \\
\hline HCV SVR, n(\%) & $10(58.8)$ & $1(20.0)$ & $9(75.0)$ & 0.10 \\
\hline EtOH Abstinence, $\mathrm{n}(\%)$ & $77(80.2)$ & $28(87.5)$ & 49 (76.6) & 0.28 \\
\hline Hemodynamic Target, n(\%) & $146(95.4)$ & $54(96.4)$ & $92(94.8)$ & 1.00 \\
\hline Stent Diameter, n(\%) & & & & 0.50 \\
\hline $8 \mathrm{~mm}$ & 99 (61.1) & $33(56.9)$ & $66(63.5)$ & \\
\hline $10 \mathrm{~mm}$ & $63(38.9)$ & $25(43.1)$ & $38(36.5)$ & \\
\hline Gradient pre-TIPS, mmHg, IQR & $14(6)$ & $14(6)$ & $14(7)$ & 0.99 \\
\hline Gradient post-TIPS, mmHg, IQR & $8(5)$ & $8(6)$ & $8(4)$ & 0.27 \\
\hline Child-Pugh Score, IQR & $8(2)$ & $8(2)$ & $8(2)$ & 0.65 \\
\hline $\mathrm{B}, \mathrm{n}(\%)$ & $138(83.6)$ & $42(72.4)$ & 96 (89.7) & \\
\hline $\mathrm{C}, \mathrm{n}(\%)$ & $27(16.4)$ & $16(27.6)$ & $11(10.3)$ & \\
\hline $\mathrm{BMI}, \mathrm{kg} / \mathrm{m}^{2}, \mathrm{IQR}$ & $22.2(6.5)$ & $23.1(7.2)$ & $22.1(6.2)$ & 0.37 \\
\hline Platelets, $\times 10^{9} / \mathrm{L}$, IQR & $125(98)$ & $124(77)$ & $129(108)$ & 0.75 \\
\hline INR, IQR & $1.3(0.2)$ & $1.3(0.3)$ & $1.3(0.3)$ & 0.26 \\
\hline Serum sodium, mmol/L, IQR & $136(8)$ & $138(7)$ & $135(7)$ & 0.09 \\
\hline Serum creatinine, $\mu \mathrm{mol} / \mathrm{L}, \mathrm{IQR}$ & $86(50)$ & $102(57)$ & $77(39)$ & $<0.01$ \\
\hline Serum bilirubin, $\mu \mathrm{mol} / \mathrm{L}, \mathrm{IQR}$ & $17(17)$ & $17(11)$ & $18(22)$ & 0.16 \\
\hline Serum albumin, g/L, SD & $34(6)$ & $34(6)$ & $34(5)$ & 0.66 \\
\hline MELD-Na, IQR & $11(8)$ & $11(8)$ & $11(8)$ & 0.99 \\
\hline L3-SMI, $\mathrm{cm}^{2} / \mathrm{m}^{2}$ IQR & NA & NA & $44.0(12.0)$ & NA \\
\hline L3-PMI, $\mathrm{mm}^{2} / \mathrm{m}^{2}, \mathrm{SD}$ & NA & NA & $510.4(216.8)$ & NA \\
\hline
\end{tabular}

Legend: Categorical variables are expressed as numbers (\%), continuous variables with a non-normal distribution are expressed as median (IQR), and continuous variables with a normal distribution are expressed as mean (SD), unless otherwise specified. ALD, alcohol-related liver disease; BMI, body mass index; HCV, hepatitis C virus; IQR, interquartile range; INR, international normalized ratio; L3-PMI, total psoas muscle area indexed for height at the third lumbar vertebrae; L3-SMI, skeletal muscle area indexed for height at the third lumbar vertebrae; MELD-Na, model for end stage liver disease with sodium; NAFLD, non-alcoholic fatty liver disease; SD, standard deviation; SVR, sustained virological response; TIPSS, transjugular intrahepatic portosystemic shunt. 
Supplemental Table 2. Baseline characteristics of patients divided with sarcopenia defined using L3-PMI .

\begin{tabular}{|c|c|c|c|c|}
\hline & $\begin{array}{l}\text { All patients } \\
(n=107)\end{array}$ & $\begin{array}{l}\text { Sarcopenia } \\
(n=28)\end{array}$ & $\begin{array}{l}\text { No Sarcopenia } \\
(n=79)\end{array}$ & $p$-value \\
\hline Age, years, SD & $55.3(9.7)$ & $56.0(9.8)$ & $55.1(9.7)$ & 0.657 \\
\hline Male sex, n (\%) & $65(60.7)$ & $17(60.7)$ & $48(60.8)$ & 1.000 \\
\hline Follow-up, months, IQR & $14.2(31.8)$ & $9.2(21.7)$ & $16.1(31.2)$ & 0.186 \\
\hline Cause of cirrhosis, $\mathrm{n}(\%)$ & & & & 0.117 \\
\hline $\mathrm{HCV}$ & $12(11.2)$ & $3(10.7)$ & $9(11.4)$ & \\
\hline ALD & $69(64.5)$ & 19 (67.9) & $50(63.3)$ & \\
\hline NAFLD & $13(12.1)$ & $1(3.6)$ & $12(15.2)$ & \\
\hline Cryptogenic & $4(3.7)$ & $3(10.7)$ & $1(1.3)$ & \\
\hline Other & $9(8.4)$ & $2(7.1)$ & $7(8.9)$ & \\
\hline HCV SVR, n (\%) & $9(75.0)$ & $2(66.7)$ & $7(77.8)$ & 1.000 \\
\hline Alcohol Abstinence, n (\%) & $49(76.6)$ & $11(68.8)$ & $38(79.2)$ & 0.498 \\
\hline Hemodynamic Target, n (\%) & $92(94.8)$ & $23(92.0)$ & $69(95.8)$ & 0.601 \\
\hline Stent Diameter, n (\%) & & & & 0.035 \\
\hline $8 \mathrm{~mm}$ & $66(63.5)$ & $22(81.5)$ & $44(57.1)$ & \\
\hline $10 \mathrm{~mm}$ & $38(36.5)$ & $5(18.5)$ & $33(42.9)$ & \\
\hline Gradient pre-TIPSS, mmHg, IQR & $14(7)$ & $15(7)$ & $14(6)$ & 0.835 \\
\hline Gradient post-TIPSS, mmHg, IQR & $8(4)$ & $8(4)$ & $9(4)$ & 0.816 \\
\hline Child-Pugh score, IQR & $8(2)$ & $8(2)$ & $8(2)$ & 0.270 \\
\hline Child-Pugh Class B, n(\%) & $96(89.7)$ & $26(92.9)$ & $70(88.6)$ & \multirow{2}{*}{0.724} \\
\hline Child-Pugh Class C, n(\%) & $11(10.3)$ & $2(7.1)$ & $9(11.4)$ & \\
\hline $\mathrm{BMI}, \mathrm{kg} / \mathrm{m}^{2}, \mathrm{IQR}$ & $22.1(6.1)$ & $18.9(4.7)$ & $22.8(5.5)$ & $<0.001$ \\
\hline Platelets, $\times 10^{9} / \mathrm{L}, \mathrm{IQR}$ & $129(108)$ & $177(145)$ & $112(102)$ & 0.009 \\
\hline INR, IQR & $1.3(0.3)$ & $1.3(0.3)$ & $1.3(0.3)$ & 0.160 \\
\hline Serum sodium, $\mathrm{mmol} / \mathrm{L}, \mathrm{SD}$ & $135(5)$ & $135(4)$ & $136(5)$ & 0.513 \\
\hline Serum creatinine, $\mu \mathrm{mol} / \mathrm{L}, \mathrm{IQR}$ & $77(39)$ & $74(51)$ & $78(38)$ & 0.528 \\
\hline Serum bilirubin, $\mu \mathrm{mol} / \mathrm{L}, \mathrm{IQR}$ & $18(22)$ & $17(16)$ & $18(26)$ & 0.399 \\
\hline Serum albumin, g/L, SD & $34(5)$ & $35(9)$ & $34(7)$ & 0.938 \\
\hline MELD-Na, IQR & $11(8)$ & $11(7)$ & $12(7)$ & 0.159 \\
\hline L3-PMI, $\mathrm{cm}^{2} / \mathrm{m}^{2}, \mathrm{SD}$ & $5.1(2.2)$ & $3.5(0.9)$ & $6.1(1.6)$ & $<0.001$ \\
\hline
\end{tabular}

Legend: Categorical variables are expressed as numbers (\%), continuous variables with a non-normal distribution are expressed as median (IQR), and continuous variables with a normal distribution are expressed as mean (SD), unless otherwise specified. ALD, alcohol-related liver disease; BMI, body mass index; HCV, hepatitis C virus; IQR, interquartile range; INR, international normalized ratio; L3-PMI, total psoas muscle area indexed for height at the third lumbar vertebrae; MELD-Na, model for end stage liver disease with sodium; NAFLD, non-alcoholic fatty liver disease; SD, standard deviation; SVR, sustained virological response; TIPSS, transjugular intrahepatic portosystemic shunt. 
Supplemental Table 3. Predictors of baseline sarcopenia using L3-PMI.

\begin{tabular}{|l|l|l|l|l|}
\hline & \multicolumn{2}{|l|}{ Univariate logistic regression } & \multicolumn{2}{l|}{ Multivariate logistic regression } \\
\hline Variables & OR $(95 \% \mathrm{Cl})$ & $\mathrm{p}$-value & OR $(95 \% \mathrm{Cl})$ & $\mathrm{p}$-value \\
\hline Age, per year & $1.01(0.97-1.06)$ & 0.65 & - & - \\
\hline BMI, per kg/m² & $\mathbf{0 . 7 7}(\mathbf{0 . 6 6 - 0 . 9 1 )}$ & $<\mathbf{0 . 0 1}$ & $\mathbf{0 . 7 8}(\mathbf{0 . 6 6 - 0 . 9 2 )}$ & $<\mathbf{0 . 0 1}$ \\
\hline Platelets & $\mathbf{1 . 0 0 ( 1 . 0 0 - 1 . 0 1 )}$ & $\mathbf{0 . 0 4}$ & $1.00(1.00-1.01)$ & 0.61 \\
\hline INR & $0.21(0.03-1.55)$ & 0.13 & - & - \\
\hline Serum creatinine & $1.00(0.99-1.01)$ & 0.57 & & \\
\hline Serum bilirubin & $0.98(0.95-1.01)$ & 0.21 & - & - \\
\hline Serum albumin & $0.98(0.90-1.06)$ & 0.62 & - & - \\
\hline MELD-Na & $0.95(0.86-1.04)$ & 0.29 & - & - \\
\hline Child-Pugh Score & $0.77(0.50-1.19)$ & 0.24 & - & - \\
\hline
\end{tabular}

Legend: $\mathrm{BMI}$, body mass index; $\mathrm{Cl}$, confidence interval; INR, international normalized ratio; MELD-Na, model for end stage liver disease with sodium; OR, odd ratio. ${ }^{\S}$ Multivariate analysis was conducted using variables with a $p$ value $<0.1$ in the univariate analysis. As a consequence, $\mathrm{BMI}$ and platelet count were the only variables entered in the MVA.

Supplemental Table 4. Predictors of de novo hepatic encephalopathy following TIPSS insertion with sarcopenia defined using L3-PMI.

\begin{tabular}{|l|l|l|l|l|}
\hline & \multicolumn{2}{|l|}{$\begin{array}{l}\text { Univariate Cox } \\
\text { regression analysis }\end{array}$} & \multicolumn{2}{l|}{$\begin{array}{l}\text { Multivariate Cox } \\
\text { regression analysis }\end{array}$} \\
\hline Variables & HR $(95 \% \mathrm{Cl})$ & $\mathrm{p}$-value & HR $(95 \% \mathrm{Cl})$ & p-value \\
\hline Age, per year & $1.00(0.96-1.04)$ & 0.95 & - & - \\
\hline BMI, per kg/m ${ }^{2}$ & $0.97(0.90-1.04)$ & 0.41 & - & - \\
\hline Platelets & $\mathbf{0 . 9 9}(\mathbf{0 . 9 9 - 1 . 0 0 )}$ & $\mathbf{0 . 0 1}$ & $\mathbf{0 . 9 9}(\mathbf{0 . 9 9 - 1 . 0 0 )}$ & $\mathbf{0 . 0 2}$ \\
\hline INR & $2.05(0.68-6.22)$ & 0.20 & - & - \\
\hline Serum creatinine & $1.00(1.00-1.01)$ & 0.68 & - & - \\
\hline Serum bilirubin & $\mathbf{1 . 0 2 ( 1 . 0 0 - 1 . 0 4 )}$ & $\mathbf{0 . 0 2}$ & - & - \\
\hline Serum albumin & $1.03(0.97-1.10)$ & 0.29 & - & - \\
\hline MELD-Na & $1.06(0.99-1.14)$ & 0.08 & $1.03(0.96-1.11)$ & 0.45 \\
\hline Child-Pugh Score & $1.09(0.77-1.53)$ & 0.63 & - & - \\
\hline Sarcopenia 3 -PMI & $1.20(0.53-2.67)$ & 0.66 & - & - \\
\hline
\end{tabular}

Legend: $\mathrm{BMI}$, body mass index; Cl, confidence interval; HR, hazard ratio; INR, international normalized ratio; L3$\mathrm{PMI}$, total psoas muscle area indexed for height at the third lumbar vertebrae; MELD, model for end stage liver disease; TIPSS, transjugular intrahepatic portosystemic shunt. ${ }^{\S}$ Multivariate analysis was conducted using variables with a $p$-value $<0.1$ in the univariate analysis. As a consequence, platelet count and MELD-Na were the only variables entered in the MVA. MELD-Na was chosen over its individual components. 
Supplemental Table 5. Predictors of mortality following TIPSS insertion with sarcopenia defined using L3-PMI.

\begin{tabular}{|c|c|c|c|c|}
\hline \multirow[b]{2}{*}{ Variables } & \multicolumn{2}{|c|}{$\begin{array}{l}\text { Univariate Cox } \\
\text { regression analysis }\end{array}$} & \multicolumn{2}{|c|}{$\begin{array}{l}\text { Multivariate Cox } \\
\text { regression analysis }\end{array}$} \\
\hline & $\mathrm{HR}(95 \% \mathrm{Cl})$ & $p$-value & $\mathrm{HR}(95 \% \mathrm{Cl})$ & $p$-value \\
\hline Age, per year & $1.04(1.00-1.07)$ & 0.04 & $1.04(1.01-1.08)$ & 0.02 \\
\hline BMI, per kg/m² & $1.02(0.95-1.08)$ & 0.61 & - & - \\
\hline Platelets & $0.99(0.99-1.00)$ & 0.01 & $0.99(0.99-1.00)$ & 0.05 \\
\hline INR & $1.38(0.42-4.51)$ & 0.60 & - & - \\
\hline Serum creatinine & $1.00(1.00-1.01)$ & 0.17 & - & - \\
\hline Serum bilirubin & $1.02(1.00-1.04)$ & 0.04 & - & - \\
\hline Serum albumin & $0.99(0.93-1.06)$ & 0.83 & - & - \\
\hline MELD-Na & $1.09(1.02-1.16)$ & 0.01 & $1.08(1.00-1.16)$ & 0.04 \\
\hline Child-Pugh Score & $1.20(0.87-1.66)$ & 0.25 & - & - \\
\hline SarcopeniaL3-PMI & $1.20(0.56-2.58)$ & 0.63 & - & - \\
\hline
\end{tabular}

Legend: $\mathrm{BMI}$, body mass index; $\mathrm{Cl}$, confidence interval; HR, hazard ratio; INR, international normalized ratio; L3$\mathrm{PMI}$, total psoas muscle area indexed for height at the third lumbar vertebrae; MELD, model for end stage liver disease. ${ }^{\S}$ Multivariate analysis was conducted using variables with a $p$-value $<0.1$ in the univariate analysis. As a consequence, age, platelet count and MELD-Na were the only variables entered in the MVA. MELD-Na was chosen over its individual components. 